\title{
THE IMPURITY CENTERS AND SOME PROBLEMS OF DIAMOND GENESIS.
}

Sobolev, E.V.

Institute of Inorganic Chemistry S.D.Ac.Sc. USSR, Novosibirsk, USSR.

The initiation of discussions dealing with the impurity centers in diamonds originates to the classical contributions of 1934 (Robertson, Fox \& Martin), who have subdivided the natural crystals into two types (I and II) with postulating the problem of the nature of differences available. The later great interest to this problem had arisen in 1959 in the classical experiments by Kaiser \& Bond, also by Smith, Sorokin, Galles \& Lasher, who had identified two structural states of impurity nitrogen - single substituting atoms (by ESR) and the non-paramagnetic associations of the substituting atoms, so-called A-centers (mass-spectrometric analysis, lattice parameter, density as compared with IR \& UV-absorption spectra). But starting with 1960 this discussion has followed a wrong trend, the idea of impurity polymorphism being refused with the wrong identification of the associative A nitrogen form as the platelets parallel to the cubic planes.

In our experiments carried out as early as 1962, also in our publications (the first published in 1964) we have chosen the trend of impurity polymorphism by proving the existence of single substituting atoms in all crystals of natural diamond (N-centers, 1964), also of A-centers being the pairs of substituting atoms (1967), nitrogen platelets - B2 centers (1965), as well as some others then unidentified nitrogen forms (1968). As late as 1971 this concept of ours was formulated by the author in a structural form which does exist at present time with some small corrections (see our review 1989). Methods applied by the author in a search of correlation between various properties of the same crystal (ESR, optical, X-ray, el. mycr., etching analysis, etc) with the quantitative basis, such as several band systems in the IR absorption spectra with the decomposition of A \& B1 bands. The principal condition of the proposed structural model of each of the centers is the lack of contradictions with none of the properties of each of the centers. Coverage of a great number of crystals (hundreds \& thounsands), among them selected carefully of much greater number has ensured high reliability of the experimental results. At present, we are familiar with over 10 nitrogen centers of various structure, five of wich are thought to be the principal for natural crystals both by their occurence and by the average and maximum nitrogen content in the specimen under study. One of the principal ones is the A-form, with nitrogen content in some cases reaching $10^{21} \mathrm{at} / \mathrm{cm}^{3}$, but the minimum recorded A nitrogen content of our specimen was $2.10^{17}$. The structure of this form as a pair of substituting nitrogen atoms in the neighbouring carbon positions follows from the over-all set of properties as well as from the nature of atbitrary paramagnetic centers formed of A-centers during particle irradiation followed by annealing or dislocational processes with a hyperfine splitting on the two nitrogen nuclei. The results obtained by Davis (1976) dealing with the symmetry fixing of A-centers using uniaxial compression methods proposed by A.A. Kaplyansky do not contradict to these data.

We have studied abundant crystals of natural diamonds from various regions (apart from the fields of USSR, we have studied those from South Africa, Australia, India, Brazil, etc) in which we have fixed $\mathrm{N}$-form, but as of secondary importance compared with the A-form. Nevertheless the correlation between them may be constant for some of the fields. By the correlation value we have separatedtwo types of fields: Yakutian \& Uralian with crystals from North Yakutia belonging to Uralian type whose mean content of N-centers is twice as high. Some of octahedra from African fields do belong to this type. Meanwhile, among the crystals of Archangelskaya Province one has fixed the mixture of two types. It is to be stressed 
that the concept that is very popular in special literature saying that the $\mathrm{N}$-centers are lacking in most of the colourless crystal of natural diamonds of highest quality is wrong; it is a consequence of the peculiarites of the apparatus applied in the experiments.

Of more complex associations of the nitrogen atoms we have separated to main group $\mathrm{N}_{3} \mathrm{~V}$ paramagnetic assemblage that are active both for absorption \& luminescence $415 \mathrm{~nm}$. The optical analogue of paramagnetic centers was identified by the author in 1969, while the modern model was published first in 1972. For a majority of natural diamond crystals the higher content of $415 \mathrm{~nm}$ centers results in increase of $\mathrm{N}$-centers versus to that of A-centers. The nitrogen contents in this form is not very high. It is much higher for B1 form (N9), that may belong according to our assumption to nitrogen segregations in octahedra planes with the three-valent substituting nitrogen atom of amino-type as a main structural element, quite like to hypothetical $\mathrm{N}_{4} \mathrm{~V}$ assemblage, a popular structural model for B1 but not $\mathrm{N} 9$ centers. The B1 (N9) centers are also active at X-rays exited blue \& cathode-luminescence with indications of concentrational saturation. This type of luminescence as well as other ones may be quenched by the A-centers, so when combined with saturation effects the X-ray ex. \& cathode luminescence of most of the natural crystals reflect at the cross-sections the quantitative distribution of A-centers. We note that the trend fixed in vast literature to assign the IR-system of BI \& UV system N9 to different centers is absolutely wrong.

Finally, the last of the principal nitrogen centers are those well known ones as early as in 60-ties, nitrogen platelats in cubic planes. Their IR-system B2 (not A) has some peculiarities connected with platelets dimension for example the maximum position of the main band etc. This experimental fact was checked many times and by many methods (for example by etching, etc.) and we must realize that Lang model of platelets but with some real structure elements such as benching from the center to periphery with the individuality of the marginal domain as a band of $1432 \mathrm{~cm}^{-1}$ etc, is quite convenient for the understanding the large family of facts.

The impurity. hydrogen can be also fixed by IR-spectra of natural diamond crystals; we hold here also the concept of impurity polymorphism as early as 1966 . The model of the main $\mathrm{C}_{2} \mathrm{H}_{2}$-center was proposed by the author in 1971; at present it has been confirmed by the recent results for the Raman spectra with indications of SERS effects etc. (see review 1989), justifying in favour of surficial localization of centers. As based on the date obtained by the authors \& those reffered in literature, these centers are most probably located at the inner surfaces of octahedral submicrocavities (viodites).

The problems of nitrogen center genesis in diamond crystals have been discussed by the author in initial contributions. We think that the viewpoint popular in special literature dealing with the origin of A-centers in the process of high-T long-time annealing from $\mathrm{N}$-centers is not correct, as it contradicts the well known of type Ib and Ia combination with type Ib included in type Ia. So the $\mathrm{N}_{2}$ centers has the growth nature, their structure is caused by the form of supply of nitrogen from the mineral-forming environment. We also regard the hydrogen $\mathrm{C}_{2} \mathrm{H}_{2}$ centers to be growth nature as they also are due to the main form of hydrogen supply. The nitrogen pairs may, in principal, be formed by only of molecular $\mathrm{N}_{2}$, but also of chemically bonded nitrogen, for exemple, to $\mathrm{CN}_{2}$, though when combined with a acetylene $\mathrm{C}_{2} \mathrm{H}_{2}$ the molecular nitrogen becomes to be the preferencial form. As for some others (principal) nitrogen \& hydrogen centers, they may be, in principal, both growth (such as $\mathrm{C}_{2} \mathrm{H}$ ) \& secondary (B1, B2) but the date obtained for natural crystals indicate that the possibility of their generation was laid probabily as a nucleus in the process of growth of each of the zones with an abrupt reduction of activation barrier for diffusion, perhaps, by the mechanism of deformational stimulation. 
Thus analysis of the structure of impurity centers in diamonds permit one to identify the two components of the mineral forming environments, such as nitrogen \& acetylene. The source of nitrogen seemed to be degassing of the Earth's Mantle, while acetylene would rather be formed by the reaction $\mathrm{CaC}_{2}+\mathrm{H}_{2} \mathrm{O} ; \mathrm{CaC}_{2}$ could be the product of the reaction of $\mathrm{CaCO}_{3}$ with metal sulphides (for diamonds these inclusions belong to the most commom ones) or oth. It is quite likely that $\mathrm{C}_{2} \mathrm{H}_{2}$ was the source of diamond carbon, though this version may be obstacled by IIa type crystals. Presence of acetylene in the natural diamond crystals permits one to understand numerous traces of dynamic processes of explosion type with crushing of crystals fixed repeatedly in the inner zoning. Fluid diffusion whose components were $\mathrm{N}_{2}$ and $\mathrm{C}_{2} \mathrm{H}_{2}$ through the enviroment may be highly inhomogeneous which may be responsible for the exceptionally inhomogeneous distribution of crystals in the source rocks.

The essential role of inhomogenethy in the processes of nitrogen diffusion was postulated by the author in 1966 in a joint paper with V.S. Sobolev.

We also believe the role of acetylene \& its products to be most probable in formation of explosion pipes at $500^{\circ} \mathrm{C}$ followed by a subduction of the cooled $\left(400-500^{\circ} \mathrm{C}\right)$ kimberlite magma into the formed emptiness. Such concepts aid in understanding numerous experimental evidence dealing with the pipes and diamonds of the principal fields, such as high preservance of crystals, often preservance of their green radiotional coat, essential diamond differentiation over the pipe cross-section, but high reproducibility both quantitatively and qualitatively by the depth and many others very commom and hardly explainable at present versions of hot kimberlite magmatism. As for the time interval between the termination of crystal growth in the upper mantle \& subduction of the quite cool kimberlite magma into the explosion pipe, the period that may be very long is to be fixed not by the impurity centers of the diamonds and included minerals but proceeding from some different experimental evidence.

ROBERTSON, R.; FOX, J.J.; MARTIN, A.E. (1934) The two types of diamond. Phyl. Trans. Roy. Soc., 232: 463-535.

KAISER, W. \& BOND, W.L. (1959) Nitrogen, a major impurity in commom type I diamond. Phys. Rev., 115: 857-863.

SMITH, W.V.; SOROKIN, T.P.; GALLES, I.L.; LASHER, G.J. (1959) Electron spin resonance of nitrogen donors in diamond. Phys. Rev., 115: 1546-1552.

DAVIES, G. (1976) The A nitrogen aggregate in diamond - its symmetry and possible structure. J. Phys. "C", Sol. St. Phys. 9: L537-L542.

SOBOREV, E.V. (1989) Harder than Diamond (sec. edit.) 190pp, Novosibirsk, Nauka (Russian). 